\title{
Nivel de actividad física y conductas sedentarias antes y durante el confinamiento a causa de la COVID-19 en adultos mexicanos Level of physical activity and sedentary behaviors before and during confinement due to COVID-19 in Mexican adults
}

\author{
*Jorge Zamarripa, *Sylvia Daniela Marroquín-Zepeda, *Oswaldo Ceballos-Gurrola, **Gabriel Flores- \\ Allende, ***Janeth Berenice García-Gallegos \\ *Universidad Autónoma de Nuevo León (México), **Universidad de Guadalajara (México), ***Universidad Autónoma \\ del Occidente (México)
}

\begin{abstract}
Resumen. La declaración por la cepa de coronavirus (SARS-CoV-2) como pandemia por la Organización Mundial de la Salud (OMS) obliga al confinamiento de millones personas, así como a modificar hábitos y estilos de vida. México es un país con altas cifras de enfermedades que se asocian con la inactividad física y el sedentarismo, las cuales provocan miles de defunciones al año. Se pretende comparar el nivel de actividad física y el tiempo dedicado a conductas sedentarias antes y durante el confinamiento a causa de la COVID-19 en una muestra de adultos mexicanos. La población objeto de estudio son 1,027 sujetos ( $52.9 \%$ mujeres y $47.1 \%$ hombres) con una mediana de 22 y promedio de 27.36 años de edad, la mayoría son estudiantes, solteros, con estudios terminados de licenciatura y nivel socioeconómico medio. Los resultados mostraron que el nivel de actividad física tiende por disminuir a causa del confinamiento en los siete días de la semana y se incrementa el tiempo destinado a comportamientos sedentarios como verTv/Video/DVD's/Netflix, descansar, hacer actividades (hobbies) cognitivos, jugar en la computadora/video juegos (no activos), escuchar música y conversar con familiares o amigos. Se confirma que el confinamiento por la COVID-19 tiene un impacto negativo en los estilos de vida de las personas durante el tiempo libre. Se requiere de innovadoras acciones para mitigar las consecuencias negativas por el confinamiento en el hogar. Palabras clave: actividad física, comportamiento sedentario, tiempo libre, confinamiento, SARS-CoV-2, México.
\end{abstract}

\begin{abstract}
The declaration of the coronavirus strain (SARS-CoV-2) as a pandemic by the World Health Organization (WHO) forces the confinement of millions of people, as well as to modify habits and lifestyles. Mexico is a country with high numbers of diseases that are associated with physical inactivity and sedentary lifestyle, which cause thousands of deaths a year. The aim is to compare the level of physical activity and the time dedicated to sedentary behaviors before and during confinement due to COVID-19 in a sample of Mexican adults. The population under study is 1,027 subjects (52.9\% women and 47.1\% men) with a median age of 22 and an average of 27.36 years of age, the majority are Latino, students, single, with completed undergraduate studies and average socioeconomic level. The results showed that the level of physical activity tends to decrease due to confinement on the seven days of the week and the time allocated to sedentary behaviors such as watching TV / video / DVD's / Netflix, resting, doing cognitive activities (hobbies), play computer / video games (not active), listen to music, and chat with family or friends increases. It confirms that COVID-19 lockdown has a negative impact on people's lifestyles during free time. Innovative actions are required to mitigate the negative consequences of home confinement.
\end{abstract}

Keywords: physical activity, sedentary behavior, leisure time, confinement, SARS-CoV-2, México.

\section{Introducción}

El esparcimiento a nivel global por la enfermedad COVID-19, a causa de una nueva cepa de coronavirus (SARS-CoV-2), provocó que el 11 de marzo de 2020 la Organización Mundial de la Salud (World Health Organization [WHO], 2021a) la declarara pandemia y problema de salud pública (Sohrabi et al., 2020). Según cifras de la OMS (WHO, 2021b) hasta enero de 2021

Fecha recepción: 20-01-21. Fecha de aceptación: 14-06-21

Jorge Zamarripa

jorge.zamarriparv@uanl.edu.mx las personas contagiadas superan los 94 millones, y poco más de 2 millones perdieron la vida.

El SARS-CoV-2 en personas infectadas (bloque de 18 - 45 años) genera síntomas leves, tales como tos seca, dolor de garganta y fiebre, estos, suelen resolverse sin tratamiento, sin embargo, en los casos más graves se pueden desarrollar complicaciones mortales tales como, falla multiorgánica, shock séptico, edema pulmonar, neumonía severa y el síndrome de distrés respiratorio agudo (Sohrabi et al., 2020). Estas consecuencias se pueden presentar principalmente en sujetos con múltiples comorbilidades, por ejemplo, la obesidad, la diabetes mellitus 2, hipertensión, inflamación crónica, entre otros (Maffetone \& Laursen, 2020). 
Específicamente en México la cifra de contagios al 19 de enero de 2021 asciende a más de 895,000 casos confirmados y 104,118 muertes. Los estados más afectados son la Ciudad de México con 123,000 casos, el Estado de México con 79,000 casos, Guanajuato con 40,000 y Nuevo León con más de 38,000 casos confirmados (Statista, 2020). Si bien los datos por las defunciones por la COVID-19 son alarmantes, las cifras por enfermedades crónico-degenerativas en ese país son de mayor medida, debido a que 90.8 millones de mexicanos presentan sobrepeso y obesidad, el 67.8 millones tienen hipertensión y 12 millones diabetes mellitus tipo 2 (Instituto Nacional de Salud Pública [INSP], 2021).

Para contrarrestar la propagación de la COVID-19 la población ha vivido aislada y en confinamiento. Durante varios meses, provocó el cierre de gimnasios, centros acuáticos, centros deportivos, parques, así como otros lugares donde las personas suelen realizar ejercicio físico o prácticar algún deporte. Esto ha supuesto que millones de personas en el mundo no puedan llevar a cabo la práctica de actividades físico-deportivas de manera habitual, causando una disminución de los niveles de actividad física a partir del confinamiento (Burtscher, Burtscher, \& Millet, 2020), impactando negativamente en la salud neuromuscular, cardiovascular y metabólica de los sujetos con implicaciones fisiológicas y fisiopatológicas (Bas, Martin, Pollack, \& Venne, 2021; Narici et al., 2020), especialmente en personas sedentarias (104\%) y con obesidad (97\%) que son más vulnerables al virus (Celis-Morales, Salas-Bravo, Y·Òez, \& Castillo, 2020). En este sentido, la OMS (2021) señala que la inactividad física es uno de los mayores problemas de salud pública en la mayoría de los países del mundo, donde México presenta además un alto porcentaje de sedentarismo.

Ammar et al. (2020) refieren que el confinamiento domiciliario por la COVID-19 tiene efectos negativos en el nivel de actividad física, además de incrementar de cinco a ocho el número de horas que las personas pasan en sus hogares sentados. Otros como Mutz y Gerke (2020) confirman la disminución en un 31\% en la práctica de deporte y ejercicio físico de tiempo libre entre alemanes, donde sólo el 27\% se mantuvo activo y apenas el 6\% incremento su nivel, son los sujetos de edad más avanzada quienes disminuyen en mayor medida la adherencia.

Entre los escasos estudios en México destaca el llevado a cabo por Rico-Gallegos et al. (2020), tuvo por objeto conocer los hábitos de actividad física y estado de salud durante el confinamiento por la COVID-19 en una muestra de 466 personas. Encuentran que el 46.35\% realiza actividad física baja, el 21.89\% actividad moderada y un $31.76 \%$ actividad física de alta intensidad; concluyen que la población presenta altos niveles de estrés. Aunque no centran en personas mayores de edad, Hall, Ochoa Martínez y Alarcon (2020) constatan que el nivel de actividad física antes y durante el distanciamiento social por la COVID-19, tiende a disminuir, principalmente, en mujeres respecto a hombres en una muestra de estudiantes de secundaria al norte del país.

Debido a que el confinamiento resulta muy aburrido y desgastante entre las personas, el interrumpir las rutinas y actividades que se realizan en el ocio para mantenerse bien (e.g., hacer ejercicio) puede incentivar algunas actividades nocivas que pueden empeorar las cosas (e.g., beber más alcohol). Además, estudios recientes han demostrado que los sintomas y el nivel de estres percibido de las personas es mas alto cuando la cantidad de semanas en confinamiento es mayor (Brooks et al., 2020; Zamarripa et al., 2020).

Al parecer no se resalta la importancia de llevar a cabo práctica de actividad física, ejercicio físico y/o deporte durante el confinamiento, se enfatiza en la importancia de mantener físicamente activo durante la pandemia, siempre que se cumpla las recomendaciones como la distancia física (Burtscher et al., 2020). Para este grupo de expertos los segmentos más vulnerables de la población son los que no logran ser físicamente activos, como el caso de las personas mayores a quienes resulta más difícil implementar actividades en el hogar. Aunque algunos estiman que las personas pueden realizar ejercicio físico de seguir algunas pautas y recomendaciones relacionadas con el distanciamiento social, la seguridad y la higiene, tanto en el interior como exterior del hogar (Dominski \& Brandt, 2020).

El presente estudio tiene como objetivo comparar el nivel de actividad física y el tiempo dedicado a conductas sedentarias antes y durante el confinamiento a causa de la COVID-19 en una muestra de adultos mexicanos.

\section{Método}

Diseño de estudio y descripción de la muestra

El presente estudio es de tipo no experimental, transversal, cuantitativo y con alcance descriptivocorrelacional. La muestra fue seleccionada a través de un muestreo no probabilistico por conveniencia. Los criterios de elegibilidad fueron los siguientes: 1) residir en México, 2) tener 18 años o más de edad, 3) otorgar 
voluntariamente su consentimiento para participar en el estudio y 4) estar en confiamiento al momento de responder el cuestionario. Para esto, se utilizó la siguiente definición de confinamiento: un estado donde se combinan estrategias para reducir las interacciones sociales como el distanciamiento social, el uso obligatorio de mascarillas, restricción de horarios de circulación, suspensión del transporte, cierre de fronteras, etcétera (Wilder-Smith \& Freedman, 2020).

Participaron 1,202 personas, de las cuales, 146 fueron eliminadas por tratarse de sujetos menores de edad, 22 por no residir en el país y 7 por información incompleta o erronea en los cuestionarios.

La muestra final quedó conformada por $n=1,027$ sujetos, donde el $52.9 \%$ son mujeres (543) y el $47.1 \%$ hombres (484) todas y todos mexicanos con una mediana de 22 y promedio de 27.36 años de edad $(D T=11.31$; rango $=18-77$ años). La mayoria de los participantes manifestaron ser estudiantes, solteros, con estudios terminados de licenciatura y se percibieron con un nivel socio-económico medio (véase Tabla 1).

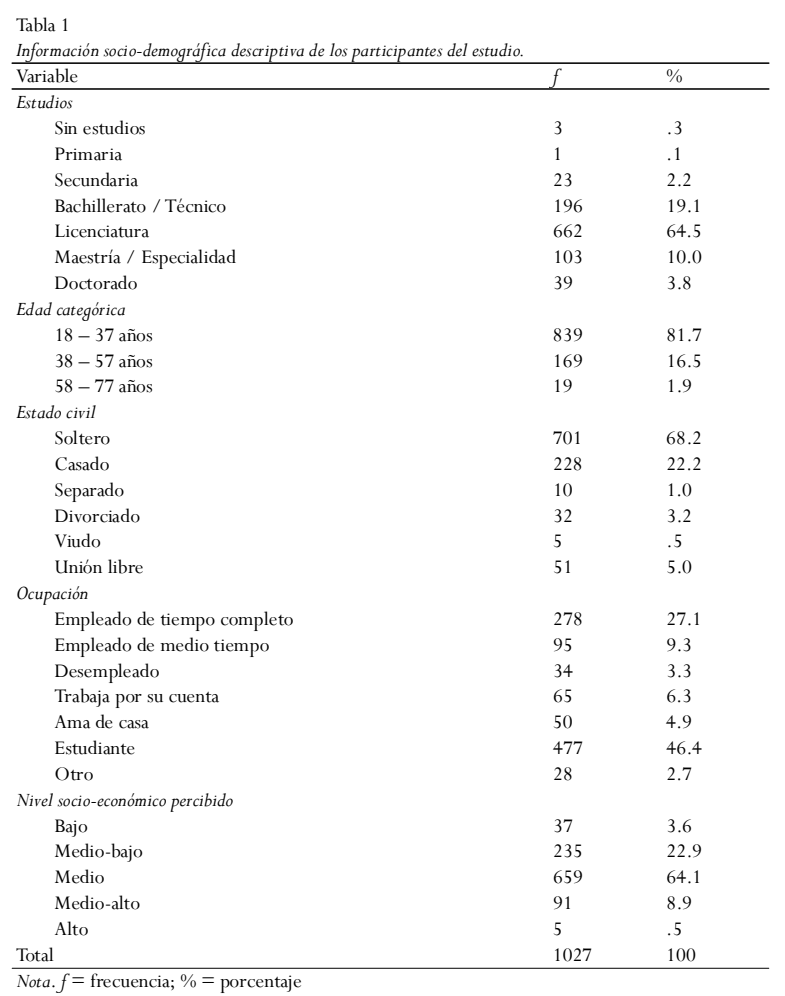

\section{Instrumentos}

Para medir la actividad física durante el tiempo libre se utilizó el Godin-Shephard Leisure-Time Physical Activity Questionnaire (LTPAQ; Godin, 2011; Godin \& Shephard, 1985). Para obtener el puntaje de actividad física de tiempo libre se siguieron las recomendaciones hechas por Godin (2011). Primero, las frecuencias semanales de actividades extenuantes, moderadas y leves se multiplican por nueve, cinco y tres, respectivamente; estos tres últimos valores corresponden a las categorías de valores de gasto energético medidos en MET's a partir de un listado de actividades. Luego, la puntuación total de la actividad física de tiempo libre semanal se calcula en unidades arbitrarias sumando los productos de los componentes separados, como se muestra a continuación: Puntuación semanal de actividad en el tiempo $=(9 \mathrm{x}$ Extenuante $)+(5 \mathrm{x}$ Moderado $)+(3$ $\mathrm{x}$ Leve). El instrumento ha presentado una adecuada fiabilidad a través de la correlación entre los datos subjetivos y objetivos relacionados con el percentil de $\mathrm{VO}_{2} \max (r=0.38, p<.001)$ y permite la correcta clasificación de individuos como aptos y no aptos del 69\% (Godin \& Shephard, 1985), así como para adultos sanos en activos e insuficientemente activos (Amireault \& Godin, 2015).

Para conocer los comportamientos sedentarios se utilizó una versión adaptada del Youth Leisure-time Sedentary Behavior Questionnaire (YLSBQ; CabanasSánchez et al., 2018), para cuantificar el tiempo que destinan las personas en una amplia gama de comportamientos sedentarios en el tiempo libre. Para el presente estudio se solicitó a los participantes que pensarán en una semana típica, antes y durante el confinamiento, sobre el tiempo promedio estimado dedicado a cada comportamiento en los días laborables de la semana (lunes a viernes) y los fines de semana (sábado y domingo) por separado. El tiempo promedio por día dedicado a cada comportamiento y categoría compuesta se calculó de la siguiente manera $=[$ (tiempo del día de la semana x 5) $+($ tiempo del fin de semana x 2)] / 7. Se obtuvo una puntuación total de tiempo sedentario sumando el tiempo informado para las 12 conductas sedentarias. El instrumento ha mostrado una buena confiabilidad test-retest y correlaciones moderadas con los datos tomados con acelerómetros en estudios previos (Cabanas-Sánchez et al., 2018), lo que sugiere un nivel razonable de validez de criterio para evaluar las conductas sedentarias.

\section{Procedimiento}

Debido a las medidas estrictas de distanciamiento social, la recopilacion de los datos se realizó de manera on-line mediante un enlace al instrumento alojado en la plataforma QuestionPro ${ }^{\circledR}$, el cual fue distribuido a través de diferentes redes sociales como Facebook ${ }^{\circledR}$, Twitter $^{\circledR}$ y Whatsapp $^{\circledR}$ durante los meses de abril, mayo y junio del 2020. En el encabezado del cuestionario se detalló el objetivo del estudio e instrucciones para res- 
ponder; se le solicitó contestar con honestidad, se le brindó informacion acerca de la confidencialidad y se solicitó su consentimiento para el uso de los datos con fines de investigacion. Sólo aquellos que dieron su consentimiento tuvieron acceso a la lectura de los instrumentos. El registro y aprobación del estudio se obtuvo del comité de revisión de ética de la coordinación de investigación de la Facultad de Organización Deportiva de la Universidad Autónoma de Nuevo León (No. REPRIN-FOD-69).

\section{Análisis de los datos}

Se realizaron análisis descriptivos de frecuencia para las variables categóricas, así como de tendencia central (media, mediana) y dispersión (desviación típica y rango) para las variables cuantitativas. Además, se realizaron análisis de normalidad para verificar la distribución de las variables cuantitativas a través de la prueba KolmogorovSmirnov con corrección Lilliefors (1967) y determinar el uso de estadística paramétrica o no paramétrica. Todo lo anterior se trabajo con el Statistical Package for the Social Sciences (SPSS) V.23.

\section{Resultados}

\section{Análisis de normalidad}

Los resultados de la prueba Kolmogorov-Smirnov resultaron significativos $(p<.001)$, lo que indica una distribución no normal de los datos en todas las variables analizadas, es decir, para los 12 comportamientos sedentarios y los tres tipos de actividad física (leve, moderado y extenuante) por separado, así como para la puntuación total de tiempo sedentario y la puntuación semanal de actividad física en el tiempo libre de antes y durante el confinamiento.

Por lo anterior, se optó por utilizar técnicas estadísticas no paramétrica, en específico, la prueba de los rangos con signo de Wilcoxon para determinar diferencias significativas $(p<.05)$ en la puntuación de la actividad física y los comportamientos sedentarios antes y durante el confinamiento y obtener una estimación de las diferencias encontradas.

\section{Comparación de actividad física en el tiempo} libre antes y durante el confinamiento

Los resultados de la prueba de los rangos con signo de Wilcoxon revela diferencias significativas entre la actividad física realizada antes y durante el confinamien- to. Como se observa en la Tabla 2, es significativa la disminución de actividad física en las tres categorías: leves, moderadas y extenuantes, así como de la puntuación total semanal de actividad física de tiempo libre durante el confinamiento. Además, la suma de rangos negativos para las actividades leves $\left(\sum^{R}-=41610\right)$, moderadas $\left(\sum^{R_{-}}=60876.50\right)$, extenuantes $\left(\sum^{R}-=\right.$ 62516) y para la puntuación total semanal de actividad física $\left(\sum^{R}-=138174.50\right)$ fueron superiores a la suma de rangos positivos de cada una de éstas $\left(\Sigma^{R}+=31161\right.$; $\sum^{R}+=36143.50 ; \sum^{R}+=32750 ; \sum^{R}+=72750.50 ;$ respectivamente), se puede afirmar que el confinamiento ha tenido un impacto negativo en la actividad física realizada durante el tiempo libre. El tamaño del efecto para estas variables oscila desde .08 hasta .21 (Tabla 2).

\begin{tabular}{|c|c|c|c|c|c|c|c|c|c|}
\hline \multirow{2}{*}{ Variable } & \multirow{2}{*}{ Situación } & \multirow{2}{*}{ Mediana } & \multirow{2}{*}{ Rango } & \multirow{2}{*}{$\begin{array}{c}\text { Rango } \\
\text { Intercuartil }\end{array}$} & \multirow[b]{2}{*}{$\mathrm{z}$} & \multicolumn{2}{|c|}{ Rangos Promedio } & \multirow{2}{*}{$\begin{array}{c}\mathrm{p} \text {-Valor } \\
\text { Wilcoxon }\end{array}$} & \multirow{2}{*}{$\begin{array}{c}\text { Tamaño } \\
\text { del Efecto }\end{array}$} \\
\hline & & & & & & Negativos & Positivos & & \\
\hline Actividades físicas leves & Antes & 2.00 & 420.00 & 5.00 & 245 & 198.14 & 18223 & 014 & 08 \\
\hline (mínimo esfuerzo) & Durante & 2.00 & 420.00 & 4.00 & & & 102.23 & .014 & \\
\hline Actividades físicas moderadas & Antes & 2.00 & 120.00 & 5.00 & & & & & \\
\hline (no exhaustivas) & Durante & 2.00 & 120.00 & 5.00 & 4.67 & 224.64 & 213.81 & $<.001$ & .15 \\
\hline Actividades físicas extenuantes & Antes & 3.00 & 211.00 & 5.00 & 5.68 & 229 & 200.92 & $<001$ & 18 \\
\hline (el corazón late rapidamente) & Durante & 2.00 & 180.00 & 5.00 & & & & & \\
\hline Puntuación semanal de & Antes & 46.00 & 1920.00 & 48.00 & 6.84 & 346.17 & 289.84 & $<.001$ & .21 \\
\hline actividad en el tiempo libre & Durante & 40.00 & 1920.00 & 48.00 & & & & & \\
\hline
\end{tabular}

Al comparar la actividad física en el tiempo libre antes y durante el confinamiento en función del género se obtuvieron diferencias significativas (con valores más altos en el antes) en las actividades físicas leves (hombres $Z=-4.192 ; p<.001$; mujeres $Z=-4.074 ; p<$ .001 ), moderadas (hombres $Z=-4.175 ; p<.001$; mujeres $Z=-2.201 ; p<.05$ ), extenuantes (mujeres $Z$ $=-2.043 ; p<.05)$ y la puntuación semanal de actividad en el tiempo libre (hombres $\mathrm{Z}=-5.162 ; p<.001$; mujeres $Z=-4.707 ; p<.001)$. Por su parte, al comparar por grupos de edad, sólo los de 18 a 37 años registraron diferencias en las actividades físicas leves $(\mathrm{Z}=$. 5.529; $p<.001)$, moderadas $(\mathrm{Z}=-4.507 ; p<.001), \mathrm{y}$ la puntuación semanal de actividad en el tiempo libre $(\mathrm{Z}=-6.270 ; p<.001)$.

Comparación de comportamientos sedentarios en el tiempo libre antes y durante el confinamiento

Respecto a los comportamientos sedentarios, la prueba de los rangos con signo de Wilcoxon también arrojó diferencias significativas antes y durante el confinamiento. En la Tabla 3 se puede apreciar una reducción significativa de tiempo destinado a viajar en transportes motorizados y hacer tarea o trabajo/estudiar con y sin computadora; por el contrario, se presenta un incremento 
significativo en el resto de actividades sedentarias entre las que destaca ver TV/video/DVD's/Netflix, descansar, hacer actividades (hobbies) cognitivos, jugar en la computadora/video juegos (no activos), escuchar musica y conversar con familiares o amigos. Asimismo, se presenta un incremento en la puntuación general total de conductas sedentarias realizadas en el tiempo libre durante el confinamiento.

La suma de rangos negativos para viajar en transportes motorizados $\left(\sum^{R}-=283101.50\right)$, hacer tarea o trabajo/estudiar sin computadora $\left(\sum^{R}-=197944\right)$ y con computadora $\left(\sum^{R}-=148093\right)$ fueron superiores a la suma de rangos positivos de cada una de éstas $\left(\sum^{R}+\right.$ $=32513.50 ; \sum^{R}+=97352 ; \sum^{R}+=121652$; respectivamente) lo que confirma un impacto negativo del confinamiento sobre este tipo de conductas sedentarias.

Por el contrario, la suma de rangos positivos fueron mayores que los negativos para el resto de conductas sedentarias como ver TV/video/DVD's/Netflix $\left(\sum^{R}+\right.$ $\left.=283273 ; \sum^{R}=75008\right)$, descansar $\left(\sum^{R}+=245858\right.$; $\left.\sum_{-}^{R}=71348\right)$, hacer actividades (hobbies) cognitivos $\left(\sum^{R}+=168354 ; \sum^{R}=66601\right)$, jugar en la computadora/video juegos (no activos) $\left(\sum^{R}+=92833 ; \sum^{R}-=\right.$ 32918), escuchar musica $\left(\sum^{R}+=143886.50 ; \sum^{R}-=\right.$ 95891.50), conversar con familiares o amigos $\left(\sum^{R}+=\right.$ 188803; $\left.\sum_{-}^{R_{-}}=134003\right)$, hablar por teléfono o enviar mensajes $\left(\sum^{R}+=142497 ; \sum^{R}-=104256\right)$, navegar en Internet por diversión $\left(\sum^{R}+=148447.50 ; \sum^{R}-=\right.$ $113278.50)$ y leer por diversión $\left(\sum^{R}+=108294 ; \sum^{R}\right.$ $=85459)$.

Asimismo, la suma de rangos positivos $\left(\mathrm{a}^{R}+=\right.$ 297746) de la puntuación total de conductas sedentarias realizadas en el tiempo libre fue mayor que la suma de rangos negativos $\left(\mathrm{a}^{R}-=214832\right)$, confirma un impacto positivo del confinamiento sobre este tipo de conductas sedentarias, es decir, el confinamiento supone el incremento en la cantidad de tiempo dedicado a comportamientos sedentarios. El tamaño del efecto para las conductas sedentarias osciló desde .07 hasta .61 (Tabla 3).

Al comparar los comportamientos sedentarios en el tiempo libre antes y durante el confinamiento en función del género se obtuvieron diferencias en ver TV/video/
DVD's/Netflix (hombres Z $=-8.409 ; p<.001$; mujeres $Z=-12.885 ; p<.001)$, jugar en la computadora/video juegos (hombres $\mathrm{Z}=-7.541 ; p<.001$; mujeres $\mathrm{Z}=$ 7.414; $p<.001)$, navegar en Internet por diversión (mujeres $\mathrm{Z}=-3.124 ; p<.05$ ), hacer tarea o trabajo/ estudiar sin computadora (hombres $Z=-5.173 ; p<$ .001 ; mujeres $Z=-7.248 ; p<.001)$, hacer tarea $o$ trabajo/estudiar con computadora (mujeres $Z=-3.124$; $p<.05$ ), leer por diversión (mujeres $Z=-2.175 ; p<$ $.05)$, sentarse y hablar con familiares o amigos (hombres $\mathrm{Z}=-2.071 ; p<.05$; mujeres $\mathrm{Z}=-4.336 ; p<.001$ ), hablar por teléfono o enviar mensajes, WhatsApp, etc. (hombres $\mathrm{Z}=-2.740 ; p<.05$; mujeres $\mathrm{Z}=-3.106$; $p$ $<.05$ ), escuchar musica (sin hacer nada más), (hombres $\mathrm{Z}=-2.081 ; p<.05$; mujeres $\mathrm{Z}=-4.763 ; p<.001)$, sentarse a descansar (hombres $Z=-8.107 ; p<.001$; mujeres $Z=-10.927 ; p<.001)$, hacer actividades (hobbies) (hombres $\mathrm{Z}=-5.285 ; p<.001$; mujeres $\mathrm{Z}=$ -8.729; $p<.001)$, viajar en transportes motorizados (hombres $\mathrm{Z}=-12.018 ; p<.001$; mujeres $\mathrm{Z}=-16.648$; $p<.001)$, y total de conductas sedentarias en el tiempo libre (mujeres $\mathrm{Z}=-4.402 ; p<.001$ ). Al comparar por grupos de edad, los de 18 a 37 registraron diferencias en todos los comportamientos sedentarios; no así, los otros grupos de edad.

\section{Discusión}

El objetivo del presente estudio era comparar el nivel de actividad física y el tiempo dedicado a conductas sedentarias antes y durante el confinamiento a causa de la COVID-19 en una muestra de adultos mexicanos.

Dado que trata de un tema sobre el cual adolece de
Tabla 3.

\begin{tabular}{|c|c|c|c|c|c|c|c|c|c|}
\hline \multirow{2}{*}{ Variable } & \multirow[b]{2}{*}{ Situación } & \multirow[b]{2}{*}{ Mediana } & \multirow[b]{2}{*}{ Rango } & \multirow{2}{*}{$\begin{array}{c}\text { Rango } \\
\text { Intercuartil }\end{array}$} & \multirow[b]{2}{*}{ z } & \multicolumn{2}{|c|}{ Rangos Promedio } & \multirow{2}{*}{$\begin{array}{l}\text {-Valor } \\
\text { Wilcoxon }\end{array}$} & \multirow{2}{*}{$\begin{array}{l}\text { Tamaño } \\
\text { del efecto }\end{array}$} \\
\hline & & & & & & Negativo & Positivos & & \\
\hline \multirow[t]{2}{*}{ VerTV/video/DVD's/Netflix. } & Antes & 21.43 & $(31.71)$ & 10.86 & \multirow{2}{*}{14.66} & \multirow{2}{*}{327.55} & \multirow{2}{*}{459.11} & \multirow{2}{*}{$<.001$} & \multirow{2}{*}{.46} \\
\hline & Durante & 26.43 & (31.71) & 11.14 & & & & & \\
\hline \multirow{2}{*}{$\begin{array}{l}\text { Jugar en la computadora/video juegos (excepto Wii, } \\
\text { Xbox kinect o similar). }\end{array}$} & Antes & 5.57 & $(31.71)$ & 10.86 & \multirow{2}{*}{9.25} & \multirow{2}{*}{199.50} & \multirow{2}{*}{276.29} & \multirow{2}{*}{$<.001$} & \multirow{2}{*}{.29} \\
\hline & Durante & 5.86 & (31.71) & 16.14 & & & & & \\
\hline \multirow{2}{*}{$\begin{array}{l}\text { Navegar en Internet por diversión (Facebook, Chat, } \\
\text { etc.). }\end{array}$} & Antes & 26.71 & $(31.71)$ & 15.57 & \multirow{2}{*}{3.14} & \multirow{2}{*}{344.31} & \multirow{2}{*}{376.77} & \multirow{2}{*}{.002} & \multirow{2}{*}{.10} \\
\hline & Durante & 26.71 & $(31.71)$ & 15.86 & & & & & \\
\hline \multirow{2}{*}{ Hacer tarea o trabajo/estudiar sin computadora. } & Antes & 20.86 & $(31.71)$ & 15.86 & \multirow{2}{*}{8.18} & \multirow{2}{*}{418.49} & 330.01 & $<001$ & 26,2 \\
\hline & Durante & 16.14 & $(31.71)$ & 20.00 & & & & & 26 \\
\hline Hacer toree o trabio/estudiar con computador & Antes & 26.14 & $(31.71)$ & 19.71 & & & & 021 & 07 \\
\hline Hacer tarea o trabajo/ estudiar con computadora. & Durante & 26.14 & $(31.71)$ & 19.43 & 2.30 & 380.70 & 352.61 & .021 & ( 07 \\
\hline Leer por diversión. & Antes & 10.86 & $(31.71)$ & 10.57 & 2.55 & 29068 & 33016 & 011 & 08 \\
\hline & Durante & 10.86 & $(31.71)$ & 15.57 & & & 330.16 & . 011 & . \\
\hline 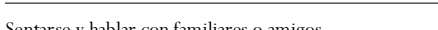 & Antes & 21.43 & $(31.71)$ & 10.86 & 417 & 38177 & 41771 & $<001$ & 13 \\
\hline Sentarse y hablar con familiares o amigos. & Durante & 21.71 & (31.71) & 15.29 & 4.17 & 381.77 & 417.71 & $<.001$ & .13 \\
\hline 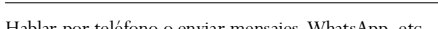 & Antes & 26.43 & $(31.71)$ & 20.00 & 3.56 & 34182 & 35893 & $<001$ & 11 \\
\hline Hablar por telerono o envar mensajes, whatsapp, et & Durante & 26.43 & $(31.71)$ & 20.29 & 3.56 & 341.82 & 358.93 & $<.001$ & .11 \\
\hline Fscuchar musica (sin hacer nada más). & Antes & 15.86 & $(31.71)$ & 11.43 & 4.57 & 327,27 & 36060 & $<001$ & \\
\hline & Durante & 16.14 & (31.71) & 15.86 & & 327.27 & 360.62 & $<.001$ & .14 \\
\hline Sentarse a desconsar (recostarse tomar upa & Antes & 16.14 & $(31.71)$ & 10.86 & 1346 & 31293 & 43285 & $<001$ & 42 \\
\hline Sentarse a descansar (recostarse, tomar una sies & Durante & 21.14 & $(31.71)$ & 10.57 & & & & & \\
\hline Hacer actividades (hobbies) cognitivos (armar & Antes & 10.57 & $(31.71)$ & 10.86 & 983 & 265.34 & 38791 & & 3. \\
\hline rompecabezas, jugar cartas, resolver crucigramas, etc). & Durante & 15.57 & (31.71) & 15.86 & & & & & \\
\hline Viajar en transportes motorizados (automóvil, autobús, & Antes & 20.57 & $(31.71)$ & 15.57 & 1939 & 42380 & 258.04 & $<001$ & 61 \\
\hline metro, motocicleta, etc.) & Durante & 10.29 & $(31.71)$ & 10.57 & & & & & \\
\hline To & Antes & 19.18 & $(31.23)$ & 6.25 & 4.46 & 46601 & 540.37 & & \\
\hline 10 & Durante & 19.77 & (28.73) & 6.17 & & & & & .1 \\
\hline
\end{tabular}


antecedentes en México y el mundo, el presente estudio resulta novedoso para el conocimiento sobre actividad física y confinamiento frente a pandemias. La limitación en el espacio para la movilidad incrementa las actividades sedentarias y se destina más tiempo sentado o acostado, con consecuencias negativas que afecta la salud y la convivencia (Chen et al., 2020). Los resultados revelan que el confinamiento como estrategía para prevenir los contagios por la COVID-19 se asocia con la disminución en el nivel de actividad física (leve, moderada o extenuante) en la población objeto de estudio.

Datos que coinciden a los obtenidos por investigadores en Alemania (Mutz \& Gerke, 2020), España (García-Tascón, Mendaña-Cuervo, Sahelices-Pinto, \& Magaz-González, 2021; Romero-Blanco et al., 2020), Slovenia (Burtscher et al., 2020) y México (Alarcón Meza \& Hall-López, 2021; Hall et al., 2020; Rico-Gallegos et al., 2020), confirman que el confinamiento por la COVID-19 se asocia con la disminución en el nivel de actividad física, dando cabida al incremento de las tasas por sedentarismo debido a los limitantes en las actividades habituales. Sin embargo, otros estudios han encontrado que el confinamiento ha supuesto una oportunidad para mejorar algunos hábitos, como la adherencia por la actividad física, aunque puede disminuir de alargar el encierro o aislamiento (Di Renzo et al., 2020).

El confinamiento por el coronavirus puede dar cabida a nuevos hábitos poco saludables, los cuales, podrían mantenerse incluso una vez concluido. Razón para considerar las recomendaciones de Margaritis et al. (2020) para quienes la combinación de una actividad física adecuada, dieta equilibrada y descanso o sueño son clave de mejores hábitos de vida.

Respecto al comportamiento antes y durante el confinamiento se observa la disminución de algunas actividades diarias como viajar en transporte motorizado y hacer tareas, estudiar o trabajar con o sin computadora, dando cabida al incremento de conductas sedentarias. Tendencia acorde a las conclusiones de algunos investigadores (Ammar et al., 2020; Kallio et al., 2018) para quienes el confinamiento, inevitablemente, induce a un estilo de vida sedentario por las largas horas que destinan a tabletas, teléfonos inteligentes y videojuegos. No se descarta un posible incremento de estrés en los sujetos por el confinamiento, como afirman Brooks et al. (2020) y Zamarripa et al. (2020) para quienes el establecer programas de actividad física puede contrarrestar estos problemas psíquicos. Así mismo, que los sujetos comiencen por presentar problemas neuromusculares, cardiovasculares y metabólicas (Narici et al., 2020).

Otro dato importante se observa en el incremento de tiempo que la población objeto de estudio destina en la semana por el confinamiento a actividades de esparcimiento como diversión, descanso y relación social, el cual, incrementó. Las limitaciones que relacionan con el contacto social físico y desplazamiento ha supuesto un incremento en el tiempo dedicado a las redes sociales (Facebook $\AA$, WhatsApp $\AA$, Twitter ${ }^{\circledR}$, Tik Tok $®$, etcétera) como un medio de comunicación, interacción y uso del tiempo libre (Tala \& Vásquez, 2020). Este tipo de conductas puede que también ocurra en otros segmentos de la población en México, como menores de edad. Por tanto, los estados de ansiedad y estrés no sólo presentan en personas adultas, sino que desde tempranas edades los efectos son negativos. El uso desmedido de las redes sociales puede causar estragos en el estado de animo y aumentar la ansiedad de los usuarios (Jeste, Lee, \& Cacioppo, 2020).

Así como se destacada los efectos nocivos frente al computadoras, tabletas, teléfonos móviles y televisión, estos apartados pueden favorecer la práctica o adherencia de ejercicio físico en el hogar, al constatar que algunos sujetos recurren a estos para seguir tutoriales y programas de entrenamiento durante la cuarentena (Piedra, 2020).

La asociación negativa entre el confinamiento a causa de la COVID-19 con el nivel de ejercicio físico puede encontrar respuestas favorables de implementar programas como el que sugieren Dominski y Brandt (2020) siempre previendo el distanciamiento físico, la seguridad y la higiene. Entendiendo que la práctica no será la misma en el hogar que al aire libre, pero tendrá como propósito fortalecer el sistema respiratorio e inmunológico, mantener la condición física y generar efectos positivos sobre la salud mental como exponen Mera-Mamián, Tabares-Gonzalez, Montoya-Gonzalez, Muñoz-Rodriguez y Monsalve-Vélez (2020).

\section{Conclusiones}

Se constata que el periodo de confinamiento como estrategia para evitar la propagación de la COVID-19 en México afectó el nivel de ejercicio físico de las personas, ya que las actividades físicas leves, moderadas y extenuantes disminuyeron durante dicho periodo. Respecto a las conductas sedentarias, se presentó un incremento durante el confinamiento en la cantidad de horas que las personas destinan al uso de las tecnologías (televisor, computadora, teléfono móvil, etc.) con fines de 
esparcimiento. Por su parte, el tiempo destinado a viajar en transportes motorizados (coche, autobus, metro, motocicleta, etc.), así como hacer tareas por cuestiones de trabajo y/o estudio con o sin computadora se redujo durante el confinamiento.

\section{Limitaciones y perspectivas futuras}

Dado que toda pandemia tiene efectos negativos para la práctica físico-deportiva se requiere de más datos para afrontar y actuar ante futuros confinamientos. Es necesario conocer la modificación de hábitos de vida, la adhesión y nivel de práctica según las variables sociodemográficas como el sexo, edad, estado civil, nivel de estudios, ocupación incluso percepción del bienestar subjetivo. También, sobre los efectos por el confinamiento en menores de edad, dado que, la suspensión de clases presenciales supuso interrumpir la activación física a través de las clases de educación física y el deporte en edad escolar, como centros de iniciación deportiva. Caso similar para estudiantes de bachillerato y grado superior.

\section{Referencias}

Alarcón Meza, E., \& Hall-López, J. (2021). Actividad física en estudiantes deportistas universitarios, previo y en el confinamiento por pandemia asociada al COVID-19 (Physical activity in university student athletes, prior and in confinement due to pandemic associated with COVID-19). Retos, 39, 572-575. doi:https: / /doi.org/ $10.47197 /$ retos.v0i39.81293

Amireault, S., \& Godin, G. (2015). The Godin-Shephard Leisure-Time Physical Activity Questionnaire: Validity Evidence Supporting its Use for Classifying Healthy Adults into Active and Insufficiently Active Categories. Perceptual and Motor Skills, 120(2), 604-622. doi:10.2466/ 03.27.PMS.120v19x7

Ammar, A., Brach, M., Trabelsi, K., Chtourou, H., Boukhris, O., Masmoudi, L., . . . Consortium, O. B. o. t. E.-C. (2020). Effects of COVID-19 Home Confinement on Eating Behaviour and Physical Activity: Results of the ECLB-COVID19 International Online Survey. Nutrients, 12(6), 1583.

Ammar, N., Aly, N. M., Folayan, M. O., Khader,Y., Virtanen, J. I., Al-Batayneh, O. B., . . . El Tantawi, M. (2020). Behavior change due to COVID-19 among dental academics - The theory of planned behavior: Stresses, worries, training, and pandemic severity. PLOS ONE, 15(9), e0239961. doi:10.1371/journal.pone.0239961

Bas, D., Martin, M., Pollack, C., \& Venne, R. (2021). The impact of COVID-19 on sport, physical activity and wellbeing and its effects on social development. UN DESA COVID-19 policy briefs. https://www.un.org/ development/desa/dspd/wp-content/uploads/sites/ 22/2020/05/PB_73.pdf

Brooks, S. K., Webster, R. K., Smith, L. E., Woodland, L., Wessely, S., Greenberg, N., \& Rubin, G. J. (2020). The psychological impact of quarantine and how to reduce it: rapid review of the evidence. The Lancet, 395(10227), 912-920. doi:10.1016/S0140-6736(20)30460-8

Burtscher, J., Burtscher, M., \& Millet, G. P. (2020). Jumping at the opportunity: Promoting physical activity after COVID-19. Scandinavian Journal of Medicine \& Science in Sports, 30(8), 1549-1550. doi:https://doi.org/10.1111/ sms. 13744

Cabanas-Sánchez, V., Martínez-Gómez, D., Esteban-Cornejo, I., Castro-Piñero, J., Conde-Caveda, J., \& Veiga, Ó. L. (2018). Reliability and validity of theYouth Leisuretime Sedentary Behavior Questionnaire (YLSBQ). Journal of Science and Medicine in Sport, 21(1), 69-74. doi:10.1016/j.jsams.2017.10.031

Celis-Morales, C., Salas-Bravo, C., Y·Òez, A., \& Castillo, M. (2020). Inactividad flsica y sedentarismo. La otra cara de los efectos secundarios de la Pandemia de COVID19. Revista médica de Chile, 148, 885-886.

Chen, P., Mao, L., Nassis, G. P., Harmer, P., Ainsworth, B. E., \& Li, F. (2020). Coronavirus disease (COVID-19): The need to maintain regular physical activity while taking precautions. Journal of Sport and Health Science, 9(2), 103-104. doi:https://doi.org/10.1016/ j.jshs.2020.02.001

Di Renzo, L., Gualtieri, P., Pivari, F., Soldati, L., Attinà, A., Cinelli, G., . . . De Lorenzo, A. (2020). Eating habits and lifestyle changes during COVID-19 lockdown: an Italian survey. Journal of Translational Medicine, 18(1), 229. doi:10.1186/s12967-020-02399-5

Dominski, F. H., \& Brandt, R. (2020). Do the benefits of exercise in indoor and outdoor environments during the COVID-19 pandemic outweigh the risks of infection? Sport Sciences for Health, 16(3), 583-588. doi:10.1007/ s11332-020-00673-z

García-Tascón, M., Mendaña-Cuervo, C., Sahelices-Pinto, C., \& Magaz-González, A.-M. (2021). La Repercusión en la calidad de vida, salud y práctica de actividad física del confinamiento por Covid-19 en España (Effects on quality of life, health and practice of physical activity of Covid-19 confinement in Spain). Retos, 42, 684-695. https: / / doi.org/10.47197/retos.v42i0.88098

Godin, G. (2011). The Godin-Shephard Leisure-Time Physical Activity Questionnaire. The Health \& Fitness Journal of Canada, 4(1), 18-22. doi:10.14288/hfjc.v4i1.82 
Godin, G., \& Shephard, R. (1985). A Simple Method to Assess Exercise Behavior in the Community. Canadian journal of applied sport sciences. Journal canadien des sciences appliquées au sport, 10, 141-146.

Hall, J., Ochoa Martínez, P., \& Alarcon, E. (2020). Actividad física, acorde a sexo, en estudiantes de secundaria antes y durante del distanciamiento social por el COVID19. Espacios, 41(42), 93-99. doi:10.48082/espaciosa20v41n42p08

INSP. (2021). Hipertensión arterial en adultos mexicanos. Retrieved from https: / /www.insp.mx/avisos/4801hipertension-adultos-mexicanos.html

Jeste, D. V., Lee, E. E., \& Cacioppo, S. (2020). Battling the Modern Behavioral Epidemic of Loneliness: Suggestions for Research and Interventions. JAMA Psychiatry, 77(6), 553-554. doi:10.1001/jamapsychiatry.2020.0027\%J JAMA Psychiatry

Kallio, P., Pahkala, K., Heinonen, O., Tammelin, T., Hirvensalo, M., Telama, R., . . . Raitakari, O. (2018). Physical Inactivity from Youth to Adulthood and Risk of Impaired Glucose Metabolism. Medicine \& Science in Sports \& Exercise, 50, 1. doi:10.1249/MSS.0000000000001555

Lilliefors, H. W. (1967). On the Kolmogorov-Smirnov Test for Normality with Mean andVariance Unknown. Journal of the American Statistical Association, 62(318), 399-402. doi:10.1080/01621459.1967.10482916

Maffetone, P. B., \& Laursen, P. B. (2020). The Perfect Storm: Coronavirus (Covid-19) Pandemic Meets Overfat Pandemic. Frontiers in Public Health, 8(135). doi:10.3389/ fpubh.2020.00135

Margaritis, I., Houdart, S., El Ouadrhiri, Y., Bigard, X., Vuillemin, A., \& Duché, P. (2020). How to deal with COVID-19 epidemic-related lockdown physical inactivity and sedentary increase in youth? Adaptation of Anses' benchmarks. Archives of Public Health, 78(1), 52. doi:10.1186/s13690-020-00432-z

Mera-Mamián, A. Y., Tabares-Gonzalez, E., MontoyaGonzalez, S., Muñoz-Rodriguez, D. I., \& MonsalveVélez, F. (2020). Recomendaciones prácticas para evitar el desacondicionamiento físico durante el confinamiento por pandemia asociada a COVID-19. Universidad y Salud, 22(2), 166-177. doi:https://dx.doi.org/ $10.22267 /$ rus. 202202.188

Mutz, M., \& Gerke, M. (2020). Sport and exercise in times of self-quarantine: How Germans changed their behaviour at the beginning of the Covid-19 pandemic. International Review for the Sociology of Sport, $O(0)$, 1012690220934335. doi:10.1177/1012690220934335

Narici, M., De Vito, G., Franchi, M., Paoli, A., Moro, T., Marcolin, G., . . . Maganaris, C. (2020). Impact of sedentarism due to the COVID-19 home confinement on neuromuscular, cardiovascular and metabolic health:
Physiological and pathophysiological implications and recommendations for physical and nutritional countermeasures. European Journal of Sport Science, 1-22. doi:10.1080/17461391.2020.1761076

Piedra, J. (2020). Redes sociales en tiempos del COVID19: El caso de la actividad física. Sociología del Deporte, 1, 41-43. doi:10.46661/socioldeporte.4998

Rico-Gallegos, C., Vargas-Esparza, G., Poblete-Valderrama, F., Carrillo-Sánchez, L., Mena-Quintana, B., ChaparroBaeza, D., \& Reséndiz-Hernández, J. (2020). Hábitos de actividad física y estado de salud durante la pandemia por COVID-19. Espacios, 41(42), 1-10. doi:10.48082/ espacios-a20v41n42p01

Romero-Blanco, C., Rodríguez-Almagro, J., Onieva-Zafra, M. D., Parra-Fernández, M. L., Prado-Laguna, M. d. C., \& Hernández-Martínez, A. (2020). Physical Activity and Sedentary Lifestyle in University Students: Changes during Confinement Due to the COVID-19 Pandemic. International Journal of Environmental Research and Public Health, 17(18), 6567.

Sohrabi, C., Alsafi, Z., O’Neill, N., Khan, M., Kerwan, A., Al-Jabir, A., . . . Agha, R. (2020). World Health Organization declares global emergency: A review of the 2019 novel coronavirus (COVID-19). International Journal of Surgery, 76, 71-76. doi:https://doi.org/ 10.1016/j.ijsu.2020.02.034

Statista. (2020). Número de casos confirmados de coronavirus (COVID-19) en México al 27 de septiembre de 2020, por entidad federativa. . Recuperado de Número de casos confirmados de coronavirus (COVID-19) en México al 27 de septiembre de 2020, por entidad federativa. Tala, Á., \& Vásquez, E. (2020). Conexión en tiempos de COVID-19. Revista médica de Chile, 148(4), 557-558. doi:https: / / dx.doi.org/10.4067/s003498872020000400557

WHO. (2021a). Coronavirus, situation in Mexico. Retrieved from https://covid19.who.int/region/amro/country/ $\mathrm{mx}$

WHO. (2021b). WHO Coronavirus Disease (COVID-19) Dashboard. Retrieved from https: //covid19.who.int Wilder-Smith, A., \& Freedman, D. O. (2020). Isolation, quarantine, social distancing and community containment: pivotal role for old-style public health measures in the novel coronavirus (2019-nCoV) outbreak. Journal of Travel Medicine, 27(2). doi:10.1093/jtm/taaa020

Zamarripa, J., Delgado-Herrada, M., Morquecho-Sánchez, R., Baños, R., de la Cruz-Ortega, M., \& Duarte-Félix, H. (2020). Adaptability to social distancing due to COVID-19 and its moderating effect on stress by gender. Salud Mental, 43(6), 273-278. doi:10.17711/sm.01853325.2020 .037 